\title{
Comparison performance of Artificial Neural Networks and Fuzzy Inference systems in forecasting precious metals price
}

\section{Case Study: Gold, Silver, Platinum and Palladium}

\author{
${ }^{1}$ AbdulSaboor Mahmoodzada, ${ }^{2}$ Suhrab Ahadi, ${ }^{3}$ Abdul Basir Mahmoodzada, \\ ${ }^{1,3}$ Faculty of Geology and Mine Engineering, Department of Mining Engineering Jawzjan University, \\ Afghanistan \\ ${ }^{2}$ Faculty Geology and Mine Engineering, Department of Geodesy Engineering Jawzjan University, Afghanistan
}

\begin{abstract}
Awareness about the price of precious metals and the correct prediction on the process of taking decision can bring facilities, and purchasing them in the global market and recognizing the specific time of dealing can cause investment. In this article comparison of the performance of Artificial Neural Networks and Fuzzy Inference Systems in predicting the price of the precious metals (Case Study: Gold, Silver, Platinum and Palladium).has been pointed. The information about each of these metals (Gold, Silver, Platinum and Palladium) is monthly considered from 1998 until 2018 including 360 data. Thus, by examining different influential variables, National Product Parameters, Time, getting higher the value of USD dollar against the Canadian dollar, global production and global reserves of precious metals are chosen as influential variables. In this research, implementation of (ANFIS) is made for the prediction model by using Artificial and Fuzzy Neural Model. Evaluation of models by using coefficient values, the average set of squares and the square root of the average set of the squares in order of the values for Gold $0.9964,0.000268 \& 0.01637$ for silver $0.987,0.000092 \& 0.0096$, for platinum $0.9976,0.000209 \& 0.01448$ and for palladium $0.99,0.0001 \& 0.01$ have been achieved. As a result, while the best predictive model for the price of gold and platinum is Artificial Neural Networks, the model of ANFIS is suggested for the silver and palladium.
\end{abstract}

Key words: Precious metals, Forecasting, Neural Network,

\section{Introduction}

Nowadays by the progress of technology and the widespread uses of computer in calculations, the smart systems of computer in artificial intelligence are becoming more and more important day by day. The techniques of intelligence artificial solve the complicated matters by imitating from the mind of human and by using the method of computing to automate the process of achieving knowledge from data [1]. These methods include the Neural Network, fuzzy interference systems and combined methods. The intelligent methods, especially when there is no prior knowledge about the data, can be so useful. The precious metals have recently attracted a lot of attention in the global market and their price is higher than the past. There are different techniques about the predictive price. The future series values which depend on the predictive sale can be obtained by secret methods. This can help the predictive sale and secret analysis which is very important for the economy and industry $[2,5]$. However, the market of precious metal is nonlinear system under the political and economic circumstances. Thus, using the classical methods for taking decision for the price of precious metals is very difficult and inefficient. We can use very efficient Neural Networks and Fuzzy Artificial Intelligence for the prediction of the price of precious metals.

There are so many advantages of using the Neural Networks; such as adaptive learning, grouping, flexibility and better generalization $[6,8]$. One of the disadvantages of the Neural 
Network is that the method results sometimes may be inexplicable. The predictive price of precious metals by the method of Neural Phase Network has so many advantages such as fast convergence, high accuracy, and strong ability of function. Analysis of price forecast results by using different kinds of methods and the result of comparing estimation can be very efficient technique for the artificial intelligence. Review of previous work in the field of price forecasting shows that the estimation has been done due to the trend of price changes [9, 10].Whereas other parameters such as the amount of reserves and availability and demand condition effect on the changes of mineral prices, Hence, in this research the trend of price changes of precious metals (includes Gold, Silver, Palladium and palatine) with reserves changes and the production rate of each metals will be checked. In addition to Neural Phase Networks, as a tool for integration to the existed data and prediction of price changes will be used in the future.

\section{Materials and Method}

Understanding the past price of precious metals and analysis of their amount and previous price which they had, is very important for the future predictive price, it is important to build an optimal model for the prediction of the price of precious metals. The factors which are effecting on the price of precious metals must be researched and studied. Selecting the best and most important factors have high importance, because knowing these kinds of factors improve the accuracy of model. In this research the price of Gold, Silver, Palatine and Palladium have been considered monthly from 1989 until 2018 for each of these precious metals 360 data are considered as well. Hence by observing other influenced variables, eventually, parameters of Gross national product parameters (GDP), time, and value of getting higher US dollars against Canadian dollars, global production and global reserves of precious metals as influential variable are chosen. The correlation and sensitivity of their price have been checked by SPSS software. After that by the assistance of Lonberk- Marco art algorithm and adjacent gradients, scaling for the neural network model and mamdani algorithms and Sekno are chosen for the model of ANFIS, after the comparison of the result of models, the predictable error will be estimated by the cooperation of statistical criteria. And the best model is chosen for the predictive price of the metals.

\subsection{Data collection}

The time series of data is a sequence of basic observation, which is recorded according to the time distance, so these sequences can be daily, weekly, monthly and yearly. The data which has been used in this research belongs to the monthly price of gold, silver, palatine, and palladium. In the selections related to the variables affecting the price of precious metals such as oil price variables, gross national product, time, interest rate, the inflation rate, the amount of precious metal reserves, and the higher rate of American dollar verse Canadian are the main reasons. In 360 relevant data sets of interval has been used for the model making from Jan 1998 until Dec 2018.Including quarterly GDP data and related to the global reserves, are the yearly, which is converted to monthly by using the Views software the table 1. Shows that the statistical information about the data and the related to the interest rate, and inflation rate and GDP which are used in this research, related to the USA. After the required data collection, the correlation between the parameters of independent and dependent variables is done and it shows that which variables have direct and indirect effect on the price of gold, silver, palatine and palladium. Therefore, the SPSS software has been used to examine the correlation coefficient of independent and related variables. 
For the forecast the price of precious metals in the table 1. Process, the correlation between the main variables was tested by Pearson correlation coefficient. The correlation coefficient indicates the intensity of the linear or non-linear relationship [(direct or inverse). The correlation range between the variables fluctuates between +1 to -1 . If the Pearson coefficient is zero, it means that there is no linear relationship between the two variables. The correlation coefficient is mention in the table 1 .

Table1. Coefficient of correlation between the variables

\begin{tabular}{|c|c|c|c|c|c|c|}
\hline \multirow{2}{*}{\multicolumn{2}{|c|}{$\left(\mathrm{R}^{2}\right)$}} & \multicolumn{5}{|c|}{ Independed variables } \\
\hline & & GDP & Date & Ex.Rate & Prduction & Reserve \\
\hline \multirow{4}{*}{ 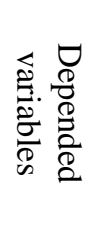 } & Gold & 0.987 & 0.995 & 0.129 & 0.683 & 0.731 \\
\hline & Silver & 0.967 & 0.995 & 0.129 & 0.879 & 0.712 \\
\hline & Platinum & 0.987 & 0.995 & 0.129 & 0.493 & 0.294 \\
\hline & Palladium & 0.987 & 0.995 & 0.129 & 0.727 & 0.234 \\
\hline
\end{tabular}

\subsection{Artificial Neural Networks}

Neural Networks are made up from simple operational element. Which operate in parallel form. These elements are inspired by the biological neuron system, and in the nature they are specified from their performance connection.

\subsubsection{The learning algorithm of Lon burg- Marko wit (LM)}

This algorithm is also called as the default MATLAB software. It is one of the most powerful types of downhill methods, as the learning of network is done very fast, and it reduces the level of existed error to the minimum. And as the same Neaten, has been designed for having no computing of not as the algorithm (LM). When the performance of function is from the square, then the function is done as followed,

$H=J^{T} J$

And the slope is measured according to the following equation:

$g=J^{T} e$

In the above equation the letter $\mathrm{J}$ represents Jacobin Matrix, that the first differential of the errors of the network that is for the bios and weighs, and the letter e is the error. The Jacobin Matrix comes through the technical computing, which has less complexity and the LM algorithm Uses the approximation for the matrix as the Neotun.

$X_{k+1}=X_{k}-\left[J^{T} J+\mu 1^{-1}\right] J^{T} e$

If the scholar of $K$ is zero, this function is used as the matrix of has in and it is changed to the Neaten methods. If the $K$ is bigger, then it is used as the slope with small steps. There for $K$ is reduced in each step and it increases when the performance of function gets increased. In this case the useable function reduces in each repetition. 


\subsubsection{Adjacent Gradient Scaling Algorithm}

Whenever the algorithm is repeated, it requires adjacent gradient algorithm. That this linear search has high cost according to the calculation.Because it must be responsive the whole network to all training inputs, which are calculated several times for the search, the adjacent gradient scale has been developed by Muller. It has been designed to avoid the time consume in linear search. So, this algorithm is more sophisticated than the few things which are described in it. But the main is to combine the model area which is used by the Lonberk Markoet, that the gradient adjacent scale approach is no longer needed. But the number of calculation in each repetition is noticeable, will reduce, because there is no linear search. Finally this algorithm is very useful for the general goals. In this research, we will study the number of hidden layers, by using the tool of (nftool) to predict the price of precious metals by using the algorithm of Lonberk Markoet for gradient adjacent scale approach. Finally, the training function will be selected with the least amount of error and the highest amount of coefficient if determination.

\subsubsection{Adaptive Neuro-Fuzzy Inference System (ANFIS)}

This method was presented for the first time by Jang in 1993, which is similar alike the Artificial Neural Network, that they take the inputs with membership function and the relevant parameters, for further explanation, the inputs and outputs are used. The main advantage of ANFIS than the regular FIS is its ability to modify its parameters than the pair of word press and output data. The structure of the Adaptive Neuro fuzzy Inference System is made of 5 layers, which is shown in the above figure. The first layer: in this layer, the process of value of function of input is made phase by using the observed phase. Each input can consist the required the function of membership. Therefore, for each membership function a specific group is defined. In this case we can have as many membership functions as we want or required. The equation of membership function is as followed:

$$
O_{l, i}=\mu_{A i}(X)
$$

In the above equation, the letter $\mathbf{X}$ stands on the input variables, and $\mathbf{A} \mathbf{i}$ represents the total of phase related to this group, and $\mu_{A i}(X)$ is usually selected as a bell, that the maximum is about 1 and the minimum is about zero( $(0)$.

$$
\mu_{A i}(x)=\frac{1}{1+\left[\left(\frac{x-c_{i}}{a_{i}}\right)^{2}\right] b_{i}}
$$

In above formula the letters (ai,bi,ci) are the total of parameters, which are known as the preliminary parameters. The $2^{\text {nd }}$ layer: it has also the whole rules between the inputs by using the phase, the product of multiplication is used to estimate the output power of each rule in the following equation: $i=1,2$

$Q_{2, i}=W_{i}=\mu_{A i}(X) X \partial \mu_{B i}(Y)$ 
The $3^{\text {rd }}$ layer: this layer is used to estimate the rule for the whole output power rate. Which is calculated as followed:

$$
W_{1}=\frac{W_{1}}{W_{1}+W_{2}} \quad \mathrm{i}=1,2,3 \ldots
$$

The $4^{\text {th }}$ layer: the product of the weights multiplied by the values of the function is calculated as following equation:

$O_{4, i}=\bar{w}_{i} f_{i}=\bar{w}_{i}\left(p_{i} x+q_{i} y+r_{i}\right)$

In the above equation the letter Wi the output of the $3^{\text {rd }}$ layer, and it is the whole parameters, which is called the parameter of result. The $5^{\text {th }}$ layer: it calculates the bottom output layer, which means that the whole signals come from the fourth layer, which is calculated according to the following equation:

Overall output $=O_{5, t}=\sum \bar{w}_{i} f_{i}=\frac{\sum w_{i} f_{i}}{\sum w_{i}}$

Neuro- Adaptive learning (ANFIS) has a similar function to neural networks. The Neural Adaptive learning provides the methods of fuzzy modeling procedure in order to get the information from the whole data. The fuzzy logic toolbox calculates the parameters of the membership function in such a way that the inference system matches to the input/ output data sets. In that case the function of ANFIS is used.

\section{Modeling}

\subsection{Design of Neural Network Models for Estimating Metal Prices}

By using the data of monthly which is used from Jan 2010 until Dec 2018 for the preparing of model, which is totally 360 data monthly. From those 360 only $70 \%$ of them is the training data, $15 \%$ of them is the validation and $15 \%$ of them is the accepted test data. In it is done through MATLAB software. In the table 2, the selected models by the Neural Network show the layers and the different neurons. The tansig, logsig and purline are used in this table as well as in the transferred function.

Table 2. The selected models by the Neural Network

\begin{tabular}{|c|c|c|c|c|c|c|}
\hline \multirow{2}{*}{$\begin{array}{c}\text { Model } \\
\text { Name }\end{array}$} & \multirow{2}{*}{$\begin{array}{c}\text { Training } \\
\text { Algorithm }\end{array}$} & \multirow{2}{*}{$\begin{array}{c}\text { Training } \\
\text { Function }\end{array}$} & \multicolumn{2}{|c|}{ Test data } & \multicolumn{2}{c|}{ Train data } \\
\cline { 4 - 7 } & & MSE & $\mathrm{R}$ & $\mathrm{MSE}$ & $\mathrm{R}$ \\
\hline Gold & trainscg & $5-10-1-1$ & 0.0002620 & 0.998313 & 0.0002681 & 0.99832 \\
\hline Silver & trainscg & $5-30-1-1$ & 0.00082716 & 0.994675 & 0.00088123 & 0.994976 \\
\hline Platinum & trainscg & $5-20-1-1$ & 0.00024115 & 0.99837 & 0.0002099 & 0.998827 \\
\hline Palladium & trainscg & $5-10-1-1$ & 0.00042373 & 0.997177 & 0.00045617 & 0.996886 \\
\hline
\end{tabular}

As we see the table 2, it shows that the best model for the gold is the model with the training function (trainscg) and the two layer topology which consist 10 neurons in the middle layer, and it can possess the transferred function (transig) and purline which consist 5 inputs and eventually it can have 1 output as well. The figure 1 . Shows that the optimal point of the 
executed model for the gold, by increasing the number of the periods from this model, is presented. In this figure, the horizontal graph shows the number of training courses and the vertical graph shows the average squares of the errors. Also the model with the training function of (trainscg) included the topology with two layers consisting 30 neurons in the middle layer is chosen as the best model for the forecast of silver, Which is pointed in figure 2. Hence, the model with the training function of (trainscg) included the topology with two layers consisting 20 neurons in the middle layer is chosen as the best model for the palatine, which is pointed in the figure 3., Also the model with the training function of (trainscg) included the topology with two layers consisting 10 neurons in the middle layer is chosen as the best model for the palladium, which is pointed in the figure 4 . The figures from 1 to 4 have been drown each of them according to the process of training, test and validation according to the MSE., As much as these three lines are closer, (as they are located in the center of the circle), the performance of the network will be much better.

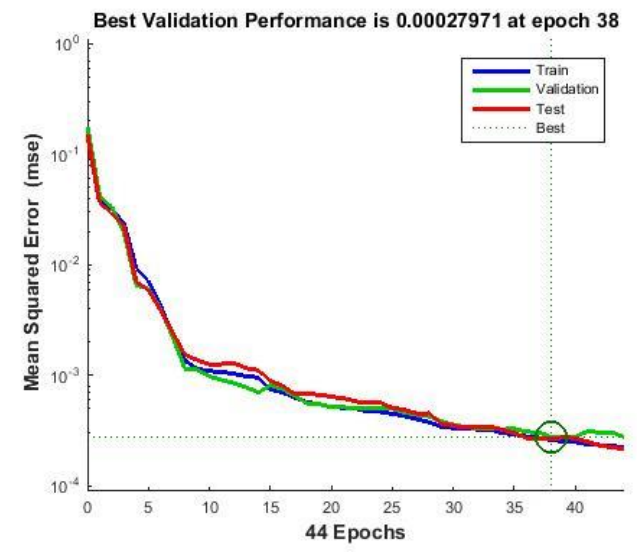

Figure 1. The trend of reducing the errors by increasing the number of periods for the gold

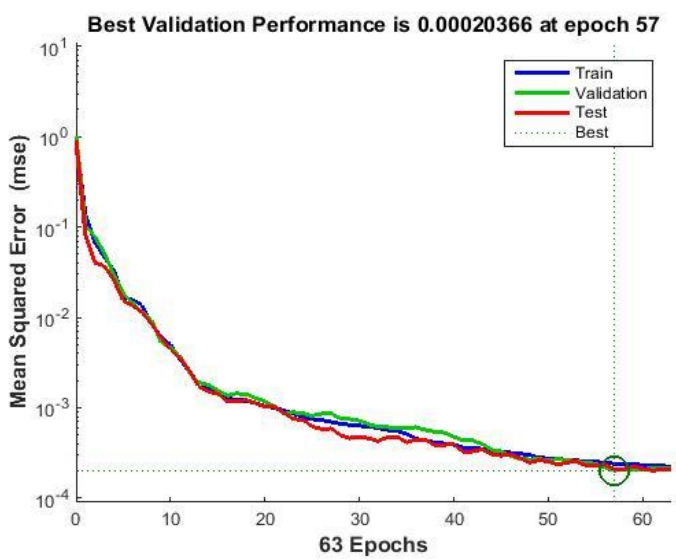

Figure 3. The trend of reducing the errors by increasing the number of periods for the palatine

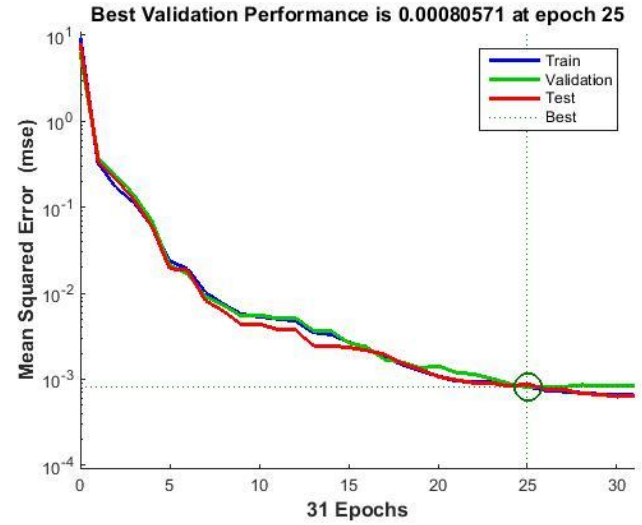

Figure 2. The trend of reducing the errors by increasing the number of periods for the silver

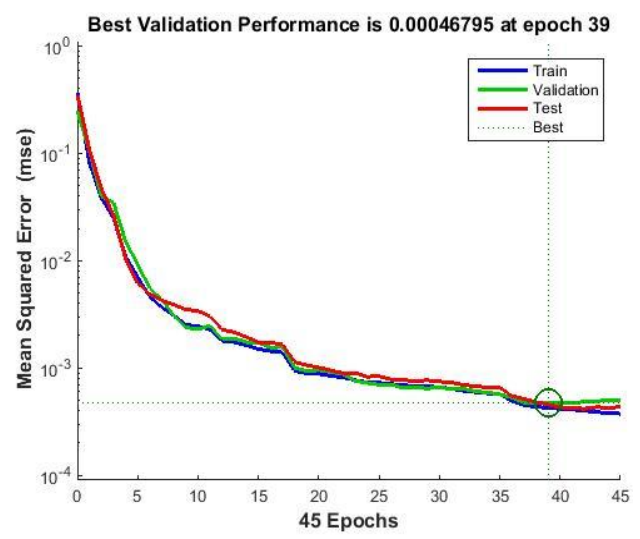

Figure 4. The trend of reducing the errors by increasing the number of periods for the palladium

These diagrams show that how much errors have occurred. As much as the amount of error is less, the performance of artificial neural network will be better. Among all of the selected models, the best is histogram of the error which is as followed:
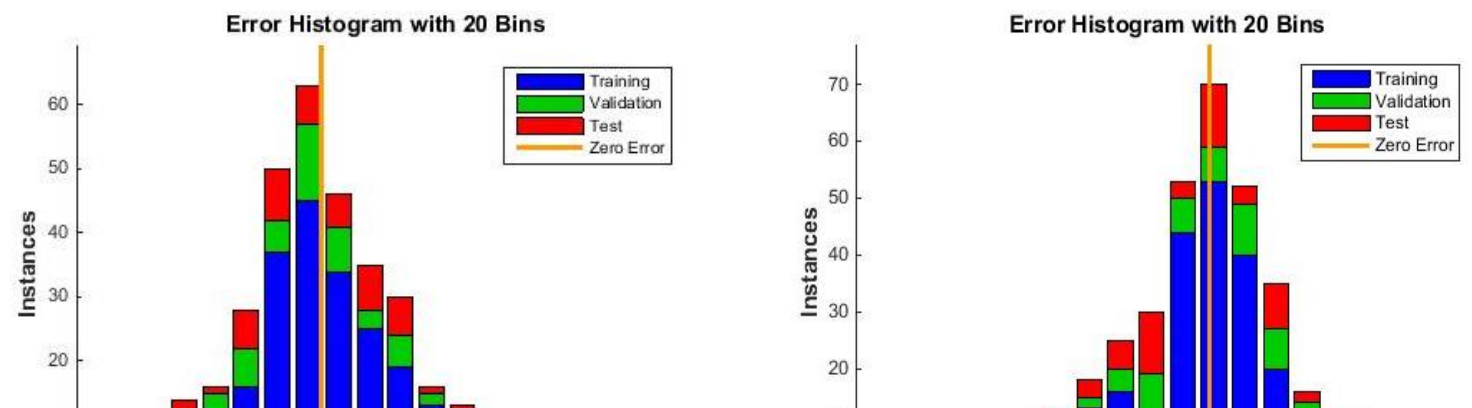


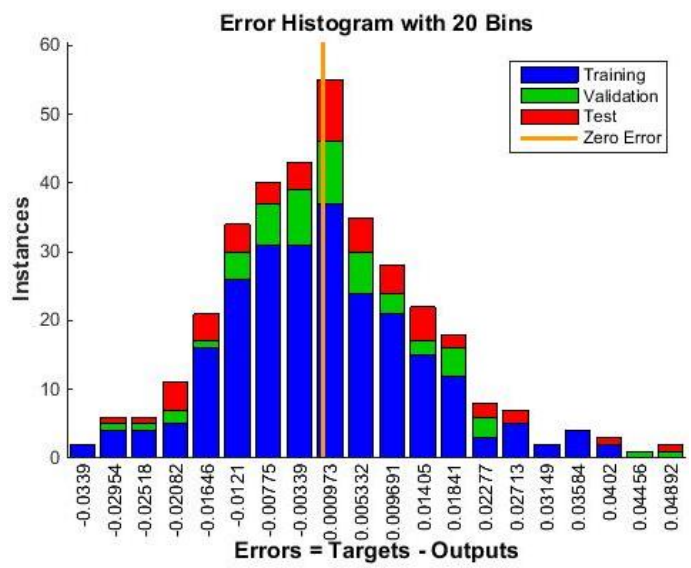

Figure 7. Error Histogram with 20 Bins for Platinum

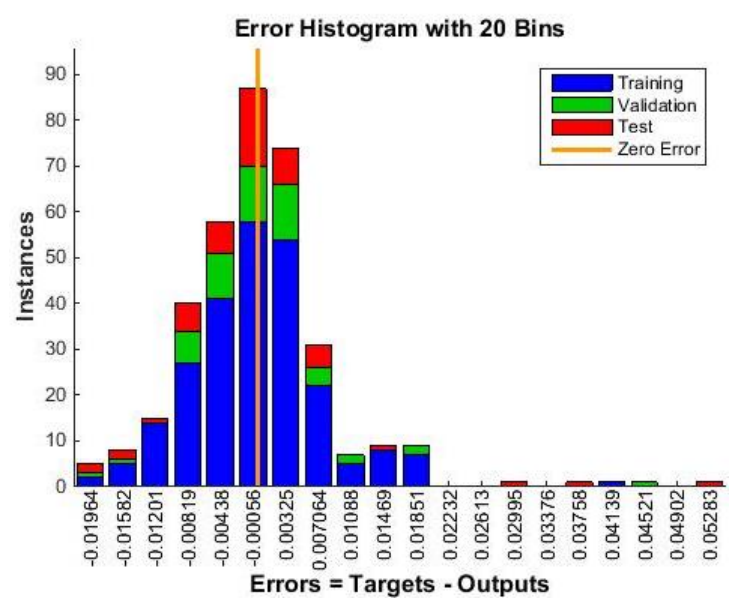

Figure 8. Error Histogram with 20 Bins for Palladium

Also the price of precious metals such as gold, silver, palatine and palladium is forecasted according to the output model of the neural network. The figures of 9, 10, 11 and 12 show the order of the comparison of the real forecast with the neural network for the gold, silver, palatine and palladium.

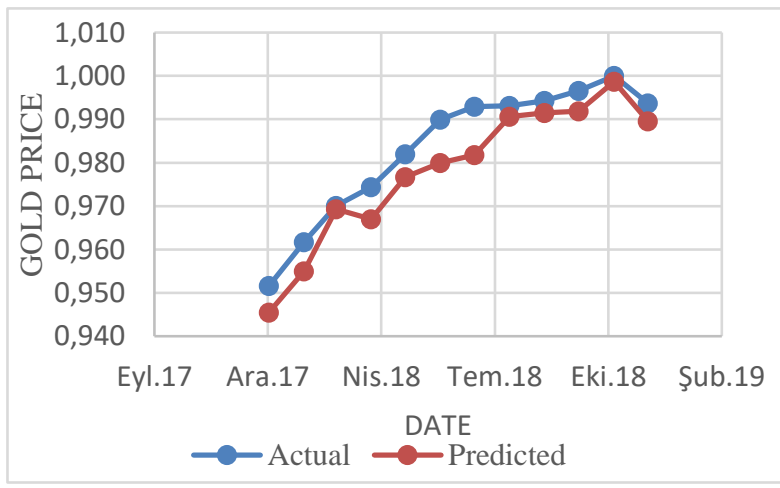

Figure 9. The comparison of the forecasted result for the neural network with the real result, gold

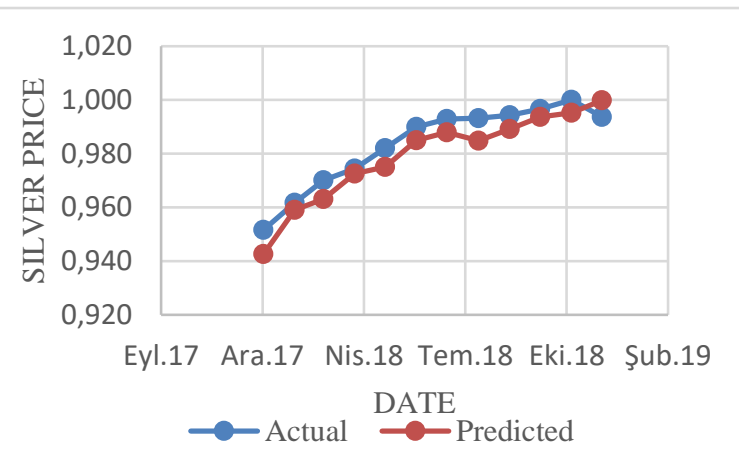

Figure 10. The comparison of the forecasted result for the neural network with the real result, silver 


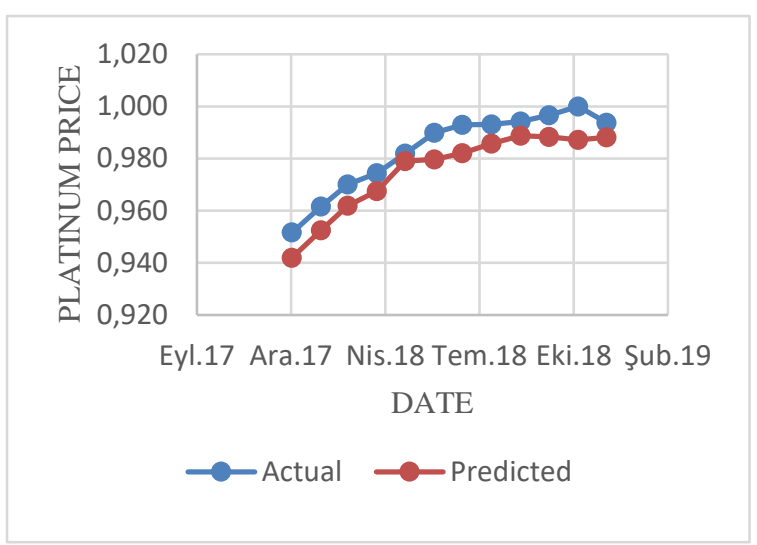

Figure 11. The comparison of the forecasted result for the neural network with the real result, platinum

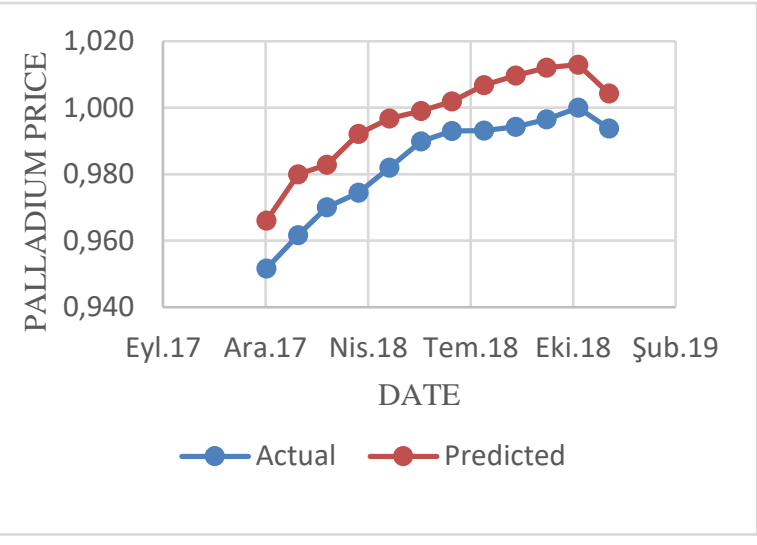

Figure 12. The comparison of the forecasted result for the neural network with the real result, Palladium

According to the calculation of output artificial neural network model, the result table 3 . Has been obtained for the gold, silver, palatine and palladium.

Table 3. The result of neural network models

\begin{tabular}{|c|c|c|c|c|c|c|}
\hline \multirow{2}{*}{ ANN } & \multirow{2}{*}{$\begin{array}{c}\text { Training } \\
\text { Algorithm }\end{array}$} & Training Function & \multicolumn{2}{|c|}{ Test data } & \multicolumn{2}{|c|}{ Train data } \\
\cline { 4 - 7 } & & RMSE & $\mathrm{R}^{2}$ & RMSE & $\mathrm{R}^{2}$ \\
\hline Gold & trainscg & $5-10-1-1$ & 0.01618 & 0.99662 & 0.01637 & 0.99664 \\
\hline Silver & trainscg & $5-30-1-1$ & 0.02876 & 0.98937 & 0.02968 & 0.98997 \\
\hline Platinum & trainscg & $5-20-1-1$ & 0.01553 & 0.99674 & 0.01448 & 0.99765 \\
\hline Palladium & trainscg & $5-10-1-1$ & 0.02058 & 0.99436 & 0.02135 & 0.99378 \\
\hline
\end{tabular}

\subsection{Design of forecasting model for the price of precious model according to the ANFIS}

In the neural fuzzy model, the training algorithm is used in order to get the parameters of the fuzzy inference system. This combination is different from the structure. Which is meant to be pointed to the methods of fuzzy adaptive training control network, intelligent control based on generalization approximation logic, adaptive neural fuzzy inference system and Neuro fuzzy system, in this research, the adaptive neural fuzzy inference system has been used for modeling of price of gold, silver, palatine and palladium, and its topic has been explained in the $2^{\text {nd }}$ chapter and it has been related to fuzzy neural system and their function. As it is expressed that the in order to predict the price of the precious metals from 360 total data have been used, from those total data 252 have been for the training data and 108 data have been for the test. The influential variables have been considered according to the gross national product, the value of USD dollar against the Canadian dollar, global production, the amount of global reserves and it is done by using the MATLAB software. The figure 13. Shows that the structure of ANFIS has been used in the modeling with the logical performance for the gold. 


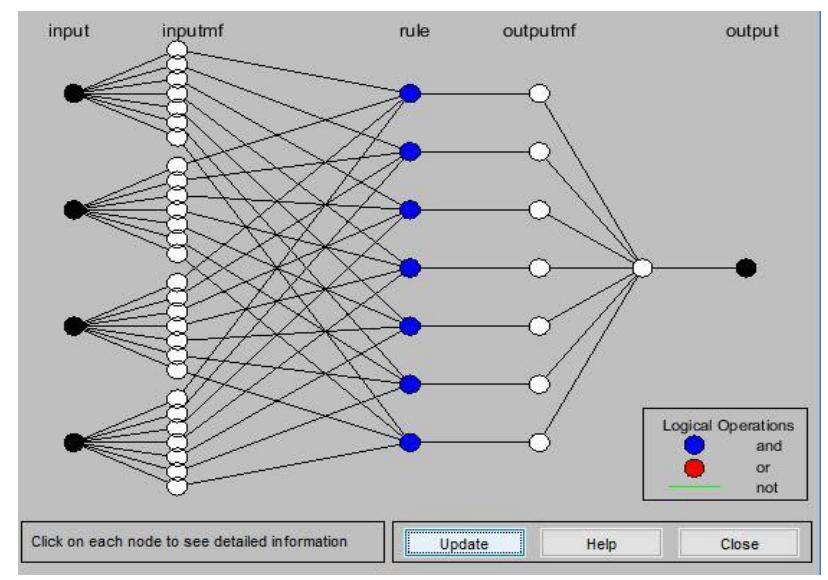

Figure 13. Shows the structure of ANFIS in the modeling with the logical performance for the gold.

Now we can show the relevant figures to the real price of forecast for the training and test of gold after achieving the input and output parameters. The real amounts of data and ANFIS have been indicated in the figure 14. Which is specified by the MSE, RMSE and R. As much as the data is matched on the line, the model will have high accuracy.
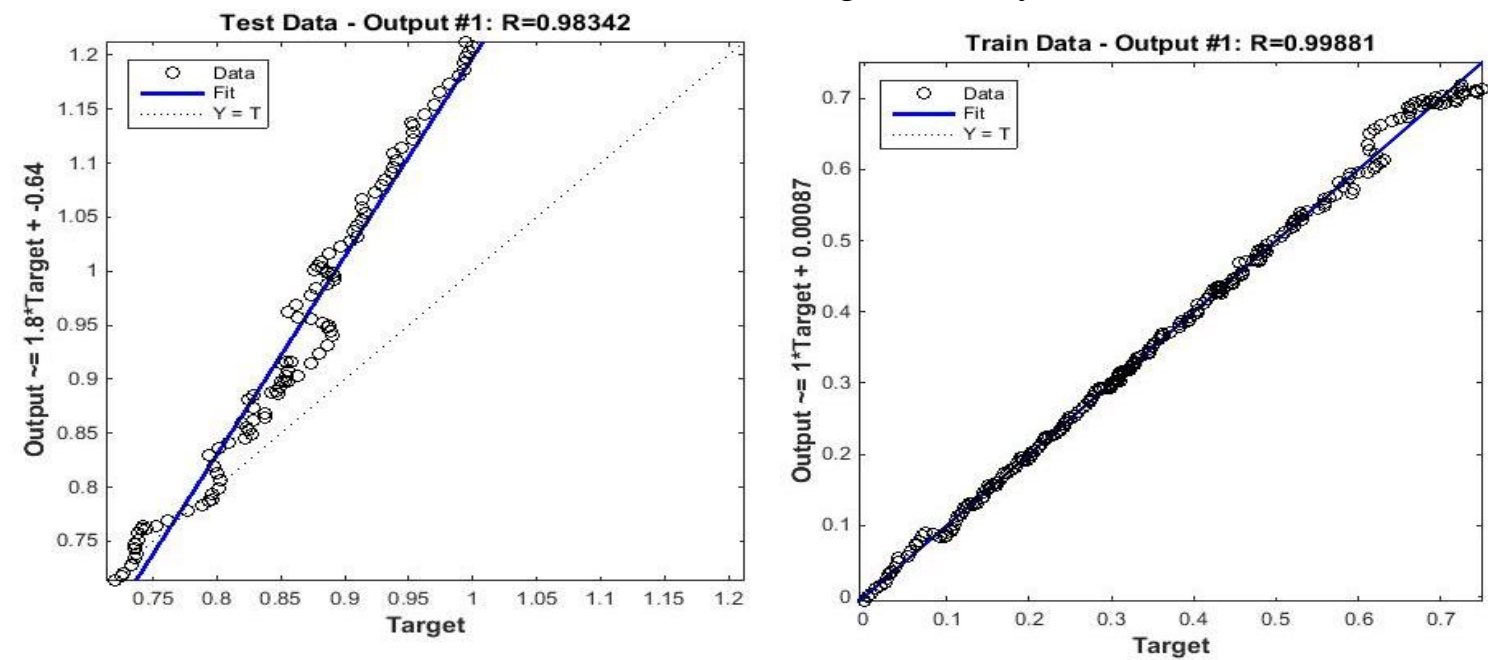

Figure 14. The forecast of the price of gold with the errors for the training and test

The following figure shows the output of ANFIS with the test data for the gold, which means that as much as these lines are attached with each other, the model of ANFIS will have high accuracy. The figure 15. Shows the real amount and predicted for the gold.
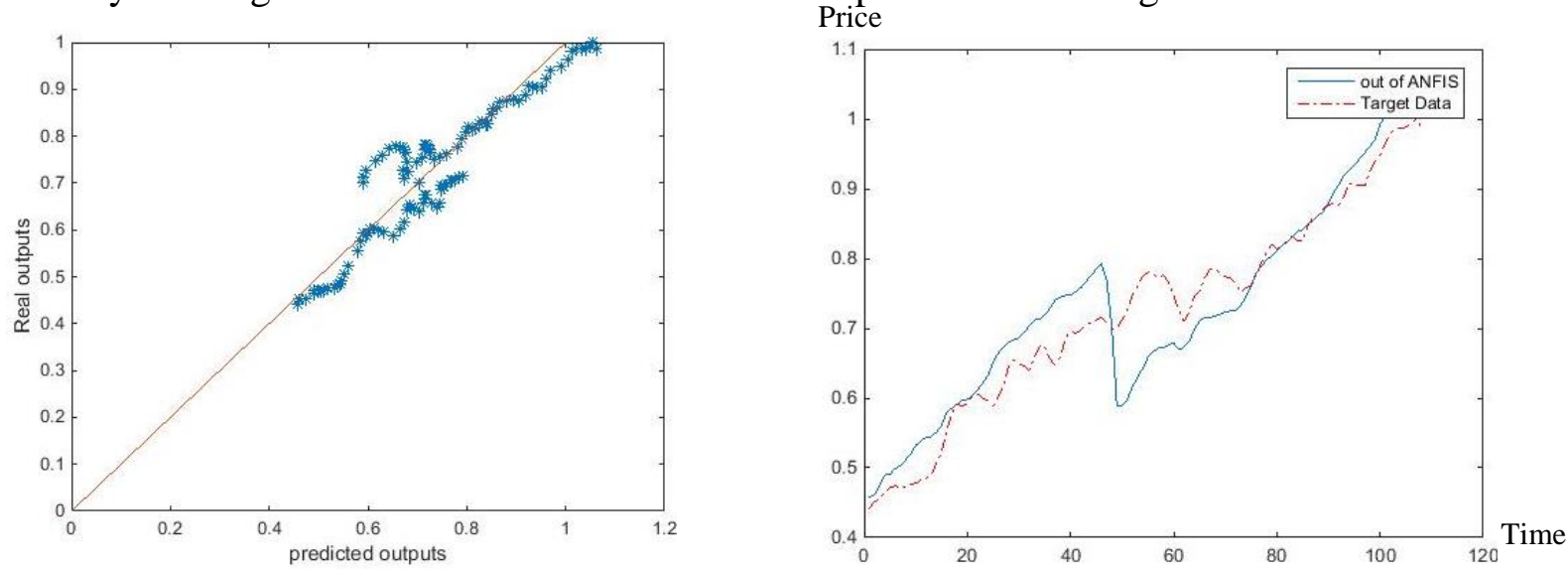

Figure 15. comparison of the output of ANFIS with the real results for the gold 
We can show the relevant figures for the forecast of real price in order to get the input and output parameters for the training data and test data. In the figure 16. The real amount of data and model have been shown, which is specified according to the RMSE, MSE and R. As much as the data is matched on the lines, the model will have high accuracy.
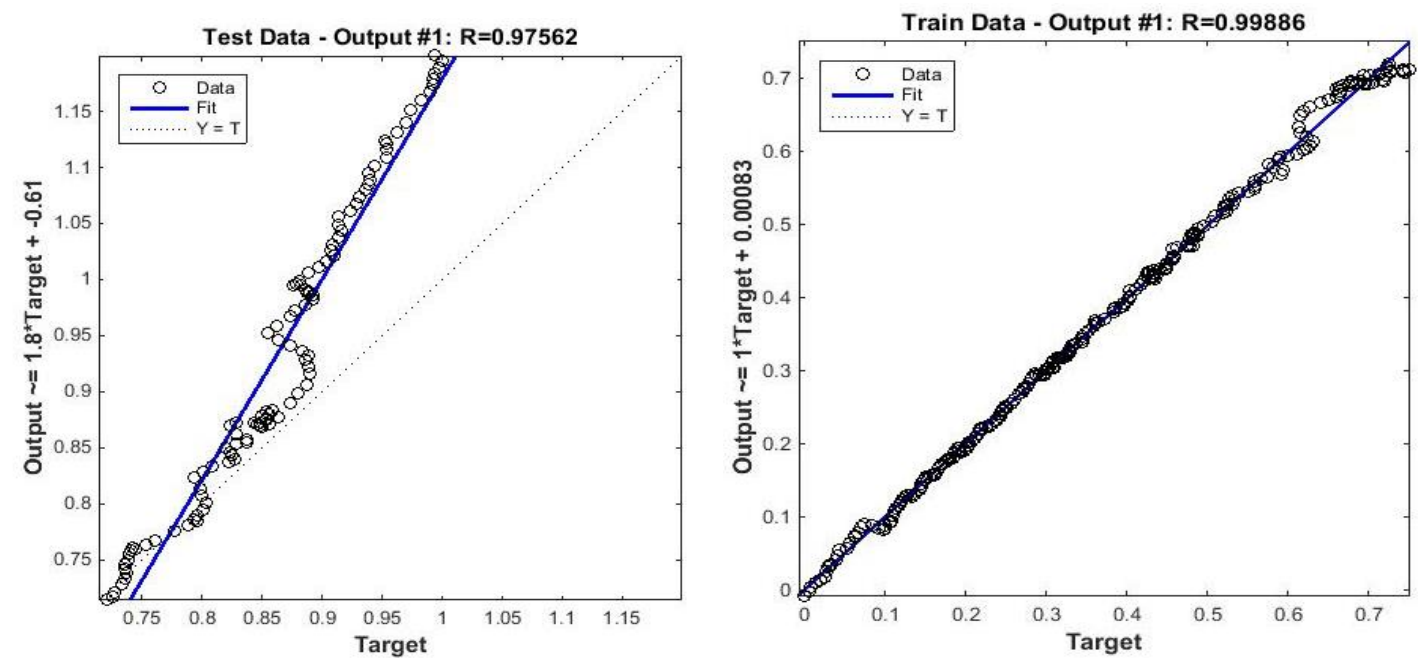

Figure 16. the forecast of the price of silver with the errors for the training data and test data

The following figure shows the output of ANFIS for the test data. Which means that as much as these lines are matched with each other, the ANFIS will have high accuracy, the figure 17. Shows the real and predicted amount for the silver.
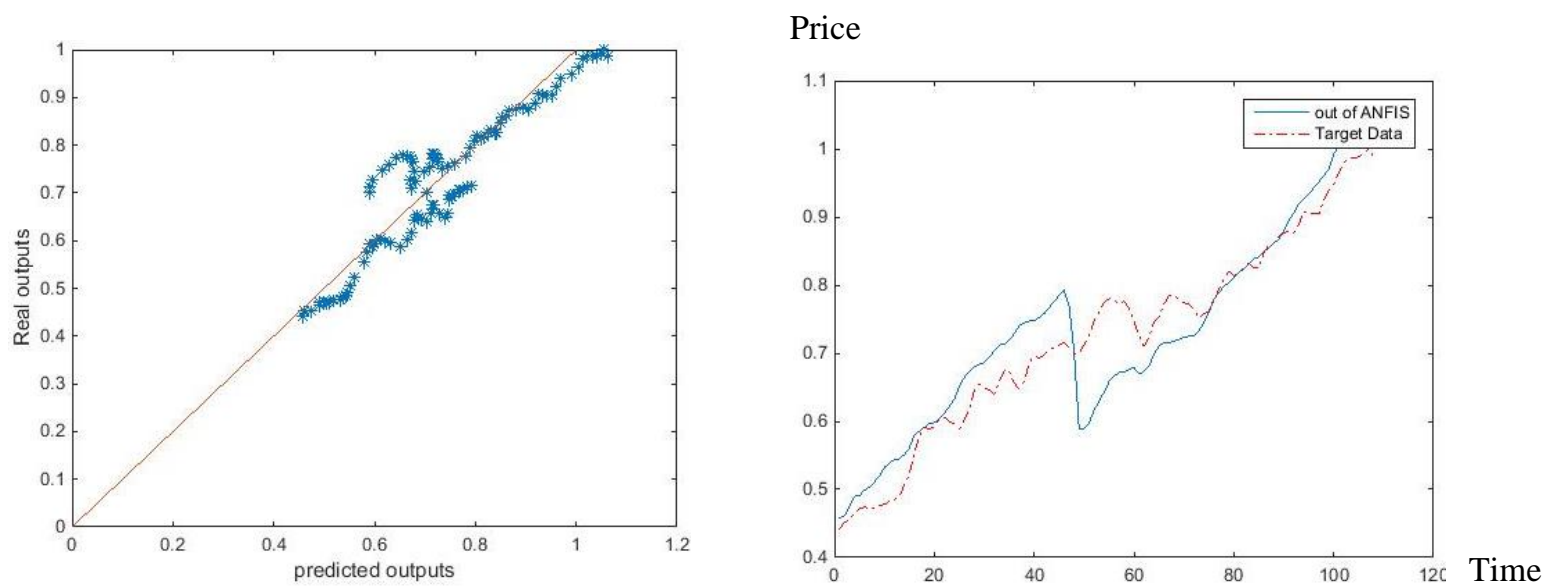

Figure 17. The comparison of the ANFIS output with real result for the silver

We can show the relevant figure for the real and predicted price for the test data and training data. In the figure 18, the real amount of data and model of ANFIS has been shown, which is specified by the RMSE, MSE and R. as much as the data is matched on the line, the model will have high accuracy. 

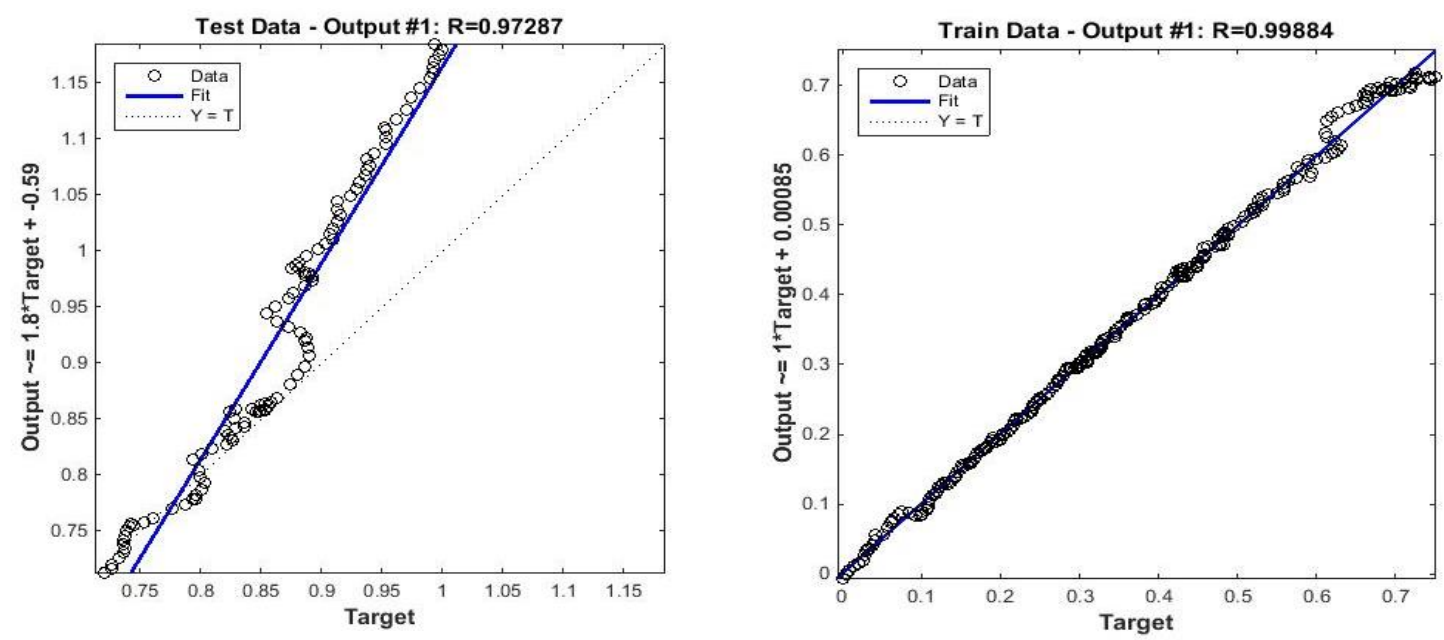

Figure 18. The predicted price of palatine with the errors for the test and training data

The following output of ANFIS figure shows the test data for the palatine, which means that as much as the lines are matched on each other, the model of ANFIS will have high accuracy, the figure 19. Shows the real and predicted amount for the palatine.

Price
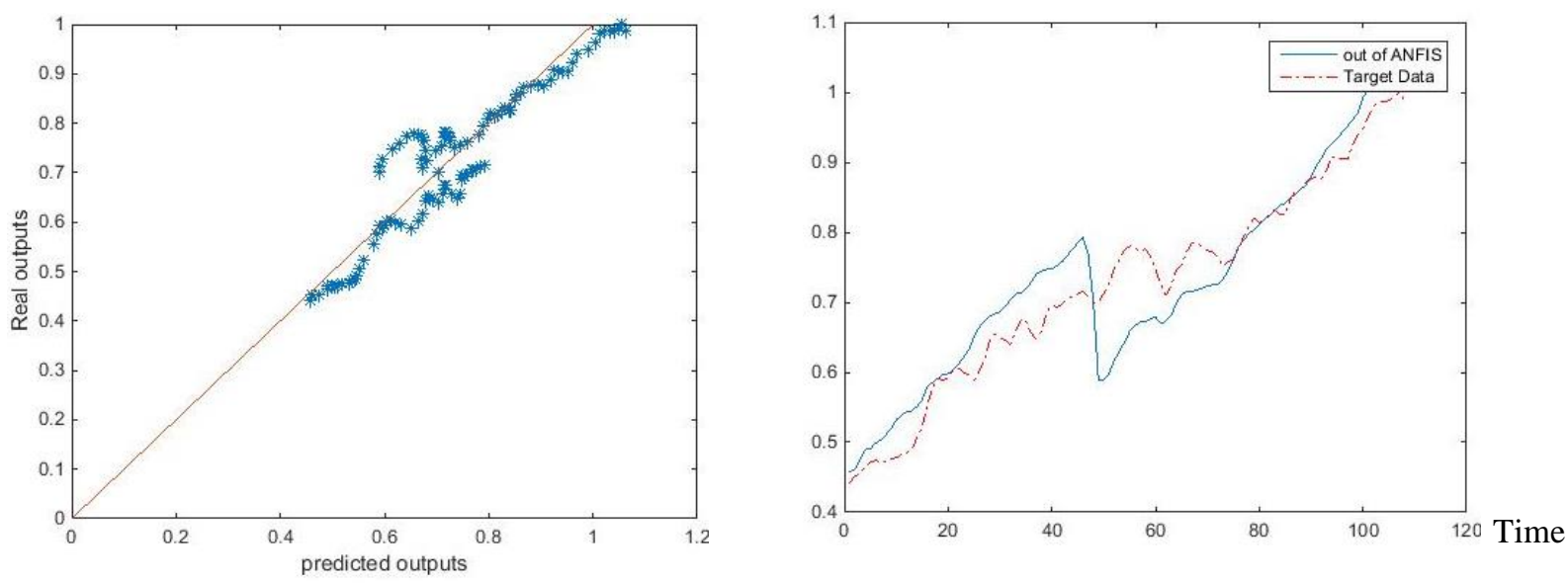

Figure 19. The comparison of output of ANFIS with the real result for the platinum

We can show the relevant figures for the real and predicted price of palladium for the test and training data. The real amount of data and the model of ANFIS have been shown in the figure 20, which is specified by RMSE, MSE and R, as much as the data is matched on the b lines, the model will high accuracy 

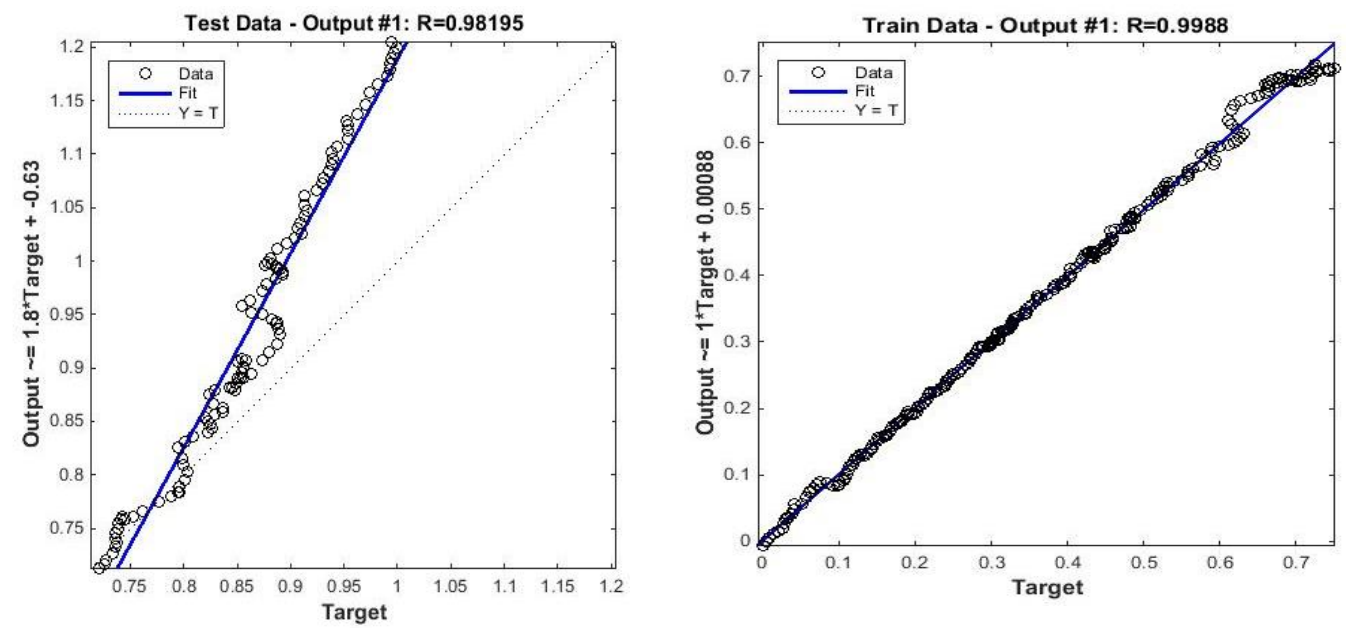

Figure 20. the forecast of the price of palladium with the errors for the test and training data

The following output figure shows the test data for the palladium, which means that as much as the lines are attached with each other, the model of ANFIS will have high accuracy, the figure of 21 shows the real and predicted amount for the palladium.
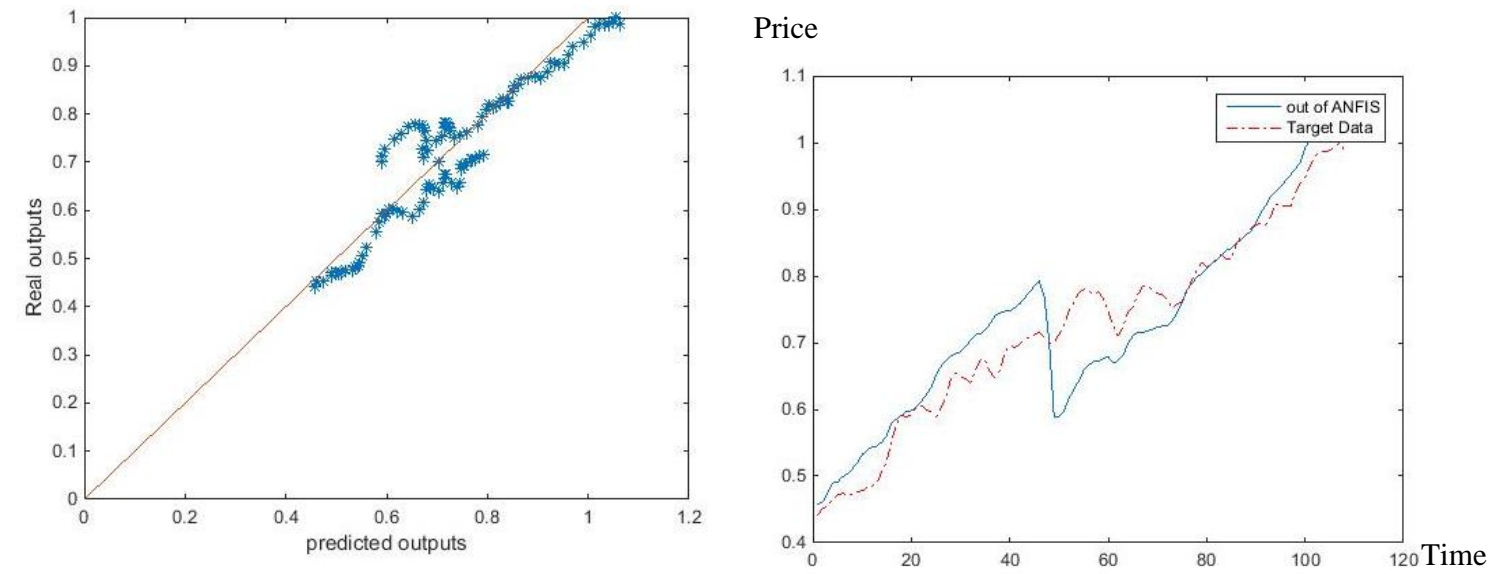

Figure 21. Comparison of the output of ANFIS with the real result for the palladium

The table 4. Shows the result of ANFIS model which is related to the each of precious metals such as (gold, silver, palatine and palladium)

Table 4. The result of ANFIS model for the precious metals

\begin{tabular}{|c|c|c|c|c|c|}
\hline \multirow{2}{*}{ NO } & \multirow{2}{*}{ ANFIS } & \multicolumn{2}{|c|}{ Train Data } & \multicolumn{2}{c|}{ Test Data } \\
\cline { 3 - 6 } & & RMSE & $\mathrm{R}^{2}$ & RMSE & $\mathrm{R}^{2}$ \\
\hline 1 & Gold & 0.0099 & 0.9993 & 0.029299 & 0.9916 \\
\hline 2 & Silver & 0.0097 & 0.99944 & 0.09601 & 0.9870 \\
\hline 3 & Platinum & 0.0098 & 0.99940 & 0.08730 & 0.9863 \\
\hline 4 & Palladium & 0.0100 & 0.9993 & 0.1005 & 0.9990 \\
\hline
\end{tabular}




\section{Results}

The table 5. Presents the general results of modeling and comparing the coefficient of determination, the average of set of squares, and the square of root of the average of the sum of the squares. The table 5. Shows that best forecasting model for gold and platinum is ANN and for silver and palladium is ANFIS.

Table5. The comparison of different methods of the price

\begin{tabular}{|c|c|c|c|c|c|}
\hline \multirow{3}{*}{ Method } & \multirow{2}{*}{ Metal Name } & \multicolumn{2}{|c|}{ Train Data } & \multicolumn{2}{c|}{ Test Data } \\
\cline { 3 - 6 } & & $\mathrm{R}^{2}$ & RMSE & $\mathrm{R}^{2}$ & RMSE \\
\hline \multirow{5}{*}{ ANN } & Gold & 0.99662 & 0.01618 & 0.99664 & 0.01637 \\
\cline { 2 - 6 } & Silver & 0.98937 & 0.02876 & 0.98997 & 0.02968 \\
\cline { 2 - 6 } & Platinum & 0.99674 & 0.01553 & 0.99765 & 0.01448 \\
\cline { 2 - 6 } & Palladium & 0.99436 & 0.02058 & 0.99378 & 0.02135 \\
\hline \multirow{5}{*}{ ANFIS } & Gold & 0.9993 & 0.0099 & 0.9916 & 0.029299 \\
\cline { 2 - 6 } & Silver & 0.99944 & 0.0097 & 0.9870 & 0.00960 \\
\cline { 2 - 6 } & Platinum & 0.99940 & 0.0098 & 0.9863 & 0.08730 \\
\cline { 2 - 6 } & Palladium & 0.9993 & 0.0100 & 0.9990 & 0.1005 \\
\hline
\end{tabular}

\section{Conclusions}

The following scenario has been presented in order to get the best model of forecasting of the price for the precious metals such as (gold, silver, platinum and palladium) The forecast of the precious metals such as (gold, silver, palatine and palladium) by using the method of Artificial Neural Network The forecasting of the price of precious metals such as (gold, silver, platinum and palladium) by using the Adaptive Neuro-Fuzzy Inference System (ANFIS) In the result the amount of coefficient of determination, average of the errors, and the average of the squares root have been compared. Eventually, the best method of forecast for the price of gold is the model of Neural Networks after the comparison and evaluating the results. And the best method for the forecast of the price of silver is the model of ANFIS, for the forecast of palatine the best method is the model of Neural Networks, and for the forecast of the price of palladium is the model of ANFIS.

\section{References}

[1] Alameer, Z., and Elaziz M. A, 2019. Forecasting gold price fluctuations using improved multilayer Perceptron neural network and whale optimization algorithm. Resources policy.

[2] Yang, J., Li, X., Liu, Q., 2017. China's copper demand forecasting based on system dynamics model: 2016-2030. Journal of Residuals Science \& Technology, Volume 14, No 3.651-

[3] Anish, C.M,.and Majhi, Babita. 2016. Hybrid nonlinear adaptive scheme for stock market prediction Using feedback FLANN and factor analysis. Jouranal of the Korean Statistical Society.

[4] Adebiyi, A.A., and Ayo, C, k. 2011.Fuzzy Neural Model with hybrid market indicators for stock Forecasting. Int. J. Electronic Finance. 
[5] Atsalakis, George S, and Balavanis, Kimon P. 2009. Forecasting stock market short-term trends Using a neuro- fuzzy based methododlgy. Expert Systems with Applecations.

[6] Amjadi N., Keynia F., and Zareipour H., 2011.Wind Power Prediction by a New Forecast Engine Composed of Modified Hybrid Neural Network and Enhanced Particle Swarm Optimization. IEEE Trans. Sustainable Energy.

[7] Antonino, Parisi. And franco parisi.,david diaz.2010.Forecasting gold price changes:Rolling and Recursive neural network models.Journal of multinational financial management.

[8] Balcilar, M., Dalkilic, A., and Wongwises, S., 2011. Artificial neural network techniques for the Determination of condensation heat transfer characteristics during downward annular flow of R134a Inside a vertical smooth tube. International Communications in Heat and Mass Transfer.

[9] Boyacioglu, Melek Acar, And Avci, Derya. 2010. An Adaptive Network - Based Fuzzy Inference System (ANFIS) for the prediction of stock market return: the case of the Istanbul Stock Exchange. Management School and Economics.

[10] Baltagi, B. H., Egger, P. and Pfaffermayr, M. 2006. A generalized spatial panel data model with Random effects. Working paper, Syracuse University, Department of Economics and Center for Policy Research.

[11] Cortez, C. A. T., Saydam, S., Coulton, J., and Sammut, C. 2017. International Journal of Mining Science and Technology Alternative techniques for forecasting mineral Commodity prices. International Journal of Mining Science and Technology.

[12] Chen, Hsin-Hung., Chen, Mingchin., and Chiu, Chun-Cheng. 2014. The Integration of Artificial Neural Networks and Text Mining to Forecast Gold Futures Prices. Communications in Statistics-Simulation and Computation.

[13] Dehghani,H., and Ataee-pour,M.2011.Determination of the effect of operating cost uncertainty on miningproject evaluation. Resources Policy

[14] Dehghani,H. , Ataee-pour,M., and Esfahanipour,A. 2014. Evaluation of the mining projects under Economic uncertainties using multidimensional binomial tree. Resources Policy.

[15] Desai, V. S., and Bharati, R. 2008. A comparison of linear regression and neural network methods For predicting excess returns on large stocks. Annals of Operations Research.

[16] Elyasiani, E., Mansur, I., and Odusami, B. 2011. Oil price shocks and industry stock returns.Energy Economics 